\title{
Monitoring drug therapy
}

Andrew W Hitchings BSc (Hons) MBBS PhD MRCP (UK) FHEA is a Senior Lecturer in Clinical Pharmacology at St George's, University of London, and an Honorary Consultant in Intensive Care Medicine at St George's University Hospitals NHS Foundation Trust. Competing interests: none declared.

\begin{abstract}
It is important to monitor drug therapy because the effects of a particular drug regimen can vary significantly between individuals. Wherever possible, therapeutic effect should be monitored using a clinical endpoint, i.e. a measure that directly reflects how the patient feels, functions or survives. In practice, it is often not feasible to use a clinical endpoint to guide therapy, particularly for preventive treatments. The next best option is to use a surrogate endpoint: a measure that changes so as to predict whether the clinical endpoint will be achieved. For a few drugs, neither a clinical nor a surrogate endpoint is available. In these instances, if the drug has a narrow therapeutic index and there is a predictable relationship between its concentration and its effects, it may be appropriate to measure its concentration in the blood. This article discusses approaches to monitoring drug therapy using clinical and surrogate endpoints, and plasma concentration monitoring. Specific guidance is provided for plasma concentration monitoring of digoxin, gentamicin, vancomycin, phenytoin, lithium and theophylline.
\end{abstract}

Keywords

Biomarkers; clinical markers; drug monitoring; drug therapy; surrogate endpoints

What is monitoring?

When we prescribe a medicine, we do so in the expectation that its benefits will outweigh its risks. Subsequently, some assessment is invariably required to confirm whether our judgement holds true for that individual. We can simply ask the patient to return if their symptoms do not improve or if they experience adverse effects. Alternatively, we can objectively assess the drug's effects. Occasionally, we measure the concentration of the drug in the blood. These are all forms of monitoring. This article will focus on monitoring the beneficial effects of drug therapy, but prescribers should also be aware of the importance of monitoring as a means of detecting early signs of adverse drug effects.

Why monitor drug therapy?

The relationship between a prescribed dosage regimen and its resultant clinical effects is complex. It can be influenced by the patient's concordance with the treatment plan (affecting the amount of drug entering the body), the manner in which the drug is handled within the body (pharmacokinetic variability) and the effect the drug has on that individual (pharmacodynamic variability). Together, these sources of variability create uncertainty over how a particular patient will respond to a particular treatment regimen. This can be resolved only by monitoring the effects of therapy in that individual.

\section{How can drug therapy be monitored?}

\section{Monitoring using clinical and surrogate endpoints}

In general, monitoring parameters are most likely to be informative if they are closely related to the clinical outcome that the treatment is intended to produce (Figure 1). Indeed, wherever possible, it is best to monitor the clinical endpoint itself. A clinical endpoint can be defined as a 'characteristic or variable that reflects how a patient feels, functions, or survives'.1 For example, when a benzodiazepine is administered to allow an interventional procedure to be performed, the clinical endpoint - sedation - is usually readily apparent. The drug dosage can be titrated to achieve the required level of sedation.

Often, however, measuring the effect of the drug on the clinical endpoint is impractical or cannot readily be used to guide therapy. This could be because the clinical endpoint is an event that cannot be detected until it is inevitable or irreversible, as is typically the case in preventive therapy (e.g. anticoagulation to reduce the risk of stroke in atrial fibrillation). Alternatively, it can be because the clinical endpoint is a delayed event, which cannot be measured until after treatment has finished. For example, the clinical endpoint in the treatment of pneumonia - cure of the infection, most reliably confirmed by the resolution of symptoms and radiographic consolidation - may not be measurable until weeks after the treatment course has ended. In these situations, we should seek to attempt to identify a suitable 
surrogate endpoint. A surrogate endpoint is a clinical variable, such as a blood test or examination finding, that does not itself affect the way the patient feels, functions or survives, but which changes in such a way as to predict whether the clinical endpoint will be achieved. Surrogate endpoints may be: -directly related to the clinical endpoint as an intermediate step in the causal pathway: for example, blood pressure (surrogate endpoint) is directly related to the risk of heart attack or stroke (clinical endpoint)

-indirectly related to the clinical endpoint; not as part of the causal pathway but changing in parallel to it: for example, correction of an abnormal body temperature (surrogate endpoint) can provide an indication of the likelihood of curing an infection (clinical endpoint).

Any biological characteristic that is objectively measured as a marker of physiological, pathological or therapeutic pathways (e.g. white cell count) can be termed a biomarker. 1 When used to measure the effect of treatment, the biomarker is acting as a surrogate endpoint. Thus, from a semantic perspective, biomarkers and surrogate endpoints can be considered related but not synonymous terms (Figure 2).

\section{Monitoring using drug concentration measurements}

A variety of factors can complicate the interpretation of plasma drug concentration, as illustrated in Figure $\underline{3}$, such that this is generally considered the monitoring parameter of 'last resort'. Criteria have been proposed to help identify drugs for which plasma concentration measurement is likely to be worthwhile. ${ }^{2}$

1. The clinical and pharmacodynamic effects of the drug are difficult to monitor $-i . e$. it is not feasible to measure the clinical endpoint directly, and no suitable surrogate endpoint exists. For example, it would clearly be inappropriate to measure the plasma concentration of a glucose-lowering agent, given that a suitable surrogate endpoint - blood glucose concentration - is readily available.

2. The relationship between plasma concentration and clinical effects is predictable - we should know the range of plasma concentrations at which there is a high probability of beneficial effects and a low risk of toxicity (the target range). For example, there is a good correlation between the plasma concentration of phenytoin and its clinical effects, with a well-defined target range. This, combined with its narrow therapeutic index (see below), makes a compelling case for monitoring plasma phenytoin concentration to guide dosage adjustment. For other antiepileptic drugs, regular plasma concentration monitoring is generally not necessary in routine practice.

3. The therapeutic index is 'narrow' - i.e. the ratio between the lowest concentration associated with toxicity, and the lowest concentration associated with benefit, is low. This means that the concentration range over which the drug is both safe and effective is narrow: there is little 'safety margin' before toxicity supervenes. This is the case for drugs such as phenytoin, lithium and gentamicin. By contrast, drugs with a broad therapeutic index (e.g. penicillins) are both safe and effective over a relatively wide concentration range. At usual dosages, therefore, the risk that the concentration will stray outside this range is much lower, making measurement unnecessary.

For drugs not fulfilling these criteria, routine measurement of plasma concentration is generally unhelpful. However, there may be exceptions. For example, whereas there is limited evidence that routine monitoring of carbamazepine concentration improves seizure control in populations, there may be a case for measuring its concentration in selected individuals. If one suspects that a suboptimal clinical response is due to non-adherence, for example, finding a very low or undetectable plasma carbamazepine concentration can be informative. Likewise, in a patient established on an effective treatment regimen, a change in circumstances that could alter the relationship between dosage and plasma concentration (e.g. use of an alternative preparation with different bioavailability) might present a reason to measure plasma concentrations to guide dosage adjustment.

The practicalities of drug concentration measurement Current recommendations with respect to drugs commonly subject to plasma concentration measurements are summarized in Table 1.

When is measurement of drug concentration indicated? Sampling may be indicated in the following circumstances: ${ }^{3}$ - There is (or, in the case of a preventive therapy, may be) inadequate clinical response, which might be attributable to subtherapeutic concentration or incomplete adherence. 
-It is difficult to determine clinically whether an adverse event is due to drug toxicity or features of the underlying condition (e.g. renal impairment occurring in a patient with sepsis may be a manifestation either of the disease or of aminoglycoside toxicity).

-There is a change in circumstances that can alter the plasma drug concentration. For example, if a patient taking lithium requires antihypertensive therapy with a thiazide diuretic or angiotensin-converting enzyme inhibitor, one should be alert to the risk of a drug-drug interaction leading to lithium accumulation. Monitoring the lithium concentration is essential in this context. Similarly, clearance of gentamicin depends on renal function; in situations where this is fluctuating, more frequent plasma concentration monitoring may be required.

When should samples be taken?

Timing in relation to doses: the concentration of drug rises and falls during the dosage interval. The interpretation of measurements made during the initial absorption and distribution phases will be complex and usually uninformative. It is therefore generally best to take samples during the elimination phase, such as at the end of the dosage interval (a 'trough' or 'pre-dose' concentration). Whatever time is selected, it is essential that it is accurately recorded with the measurement request, otherwise interpretation will be impossible.

Timing in relation to the start of the treatment regimen: following the introduction of a medicine, the amount of drug in the body will accumulate. At some point, provided the situation remains stable, a state of equilibrium will be reached at which the amount of drug administered in a given period is equal to the amount of drug eliminated during that period. This is termed steady state. The time taken to reach steady state depends on the half-life $(t / 2)$ of the drug. A good rule of thumb is that steady state will be achieved five half-lives after the introduction of the drug or any change to the dosage regimen.

For example, the antibiotic vancomycin has a half-life of approximately 6 hours. After starting treatment, it will take approximately 30 hours $(5 \times 6$ hours) for steady state to be achieved (Figure $4 a)$. Only at this point can the plasma concentration associated with that dosage regimen be reliably assessed. Likewise, if the dosage is changed, it will take another 30 hours before a new steady state is reached.

There are, however, circumstances in which it can be useful to measure concentrations before steady state is reached: ${ }^{3}$

- Concentrations approximating steady state can be achieved sooner if a loading dose has been given (Figure 4b).

-After starting a drug with a narrow therapeutic index and a long half-life, it may be appropriate to measure its concentration before steady state is achieved, as an early indicator of whether the steadystate concentration is likely to exceed the target range. This can provide an opportunity to adjust the dosage before toxicity occurs.

- If drug concentration is being measured to aid the diagnosis of toxicity, it can be measured irrespective of whether steady state has been achieved.

The concept of steady state is meaningful only if doses are administered sufficiently frequently that the previous dose has not been completely cleared before the next dose is given. If the dosage interval is substantially longer than the half-life of the drug (typically $\geq 5 \times t / 2$ ), the regimen may be better visualized as recurring single doses. For example, in a patient with normal renal function, gentamicin has a half-life of approximately 2 hours. For most indications, gentamicin is administered once daily. In this scenario, there is no meaningful accumulation between doses, and the pre-dose (trough) concentration should be negligible (Figure 4c). By measuring the trough gentamicin concentration, one can identify those patients in whom gentamicin clearance is reduced (invariably due to impaired renal function), and who might therefore be exposed to accumulating gentamicin concentrations if the dose and/or dosage interval is not adjusted.

\section{How do the results guide treatment?}

Interpreting the target range: the target range is derived from population studies. Natural inter-individual variation dictates that there will be people who do not derive therapeutic benefit within the population target range, and others who experience toxicity below the population toxicity range. Moreover, the range may need to be interpreted in light of other variables. For example, phenytoin is heavily protein-bound, but only the unbound drug exerts an effect. If the measured concentration includes both the unbound and 
protein-bound fractions (i.e. it is a 'total' concentration), then, in the presence of hypoalbuminaemia, a low total concentration could be associated with a therapeutic or toxic concentration of unbound drug. ${ }^{4}$ It is therefore more informative to measure the unbound fraction specifically, but if this facility is unavailable, a mathematical correction can be applied to allow the total concentration to be assessed against the standard target range (Table 1).

The concept of diminishing returns: as discussed in 'Pharmacodynamics for the prescriber' ( $p p x x x-$ $x x x$ of this issue), dose-response curves classically take a sigmoidal form. Dosage increases within the log-linear portion of the curve might reasonably be expected to lead to a discernible increase in response. However, as the concentration rises further, the curve plateaus. Increases in dosage now produce progressively less incremental change in therapeutic effect. By contrast, there may be a marked increase in the risk of toxicity.

Frequency and magnitude of dosage adjustments: the appropriate frequency of measurements depends to a great extent on the stability of the patient's condition. During times of stability, excessively frequent monitoring can simply reveal fluctuations that are an inherent part of clinical measurement (due to both biological and analytical variability). This can encourage prescribers to make changes that are not warranted. ${ }^{5}$ Likewise, excessive changes in dosage can lead to what has been described as the 'pingpong' phenomenon. ${ }^{5}$ The combined effects of overzealous changes in dosage, too early a remeasurement of the monitoring variable, and natural fluctuations, can lead to a series of treatment changes that generate increasing instability. It is generally advisable to make only small changes at a time, remeasuring only once a new steady state has been attained.

\section{Key points}

-Drug therapy should be monitored due to the potential for inter-individual variability in drug response -Wherever possible, drug therapy should be monitored using a clinical endpoint - a characteristic or variable that reflects how a patient feels, functions or survives

-When it is not possible to use a clinical endpoint to guide therapy, a surrogate endpoint can be used - a characteristic or variable that changes in such a way as to predict whether the clinical endpoint will be achieved

-Measurement of plasma concentrations is indicated for only a few drugs - those in which effects of the drug are difficult to measure, the relationship between plasma concentration and clinical effects is predictable or the therapeutic index is narrow

-When measuring the plasma concentration of a drug, usually take the sample at steady state (at least five half-lives after starting the dosage regimen), and always record the time of the sample in relation to the last dose 


\section{Figure 1}

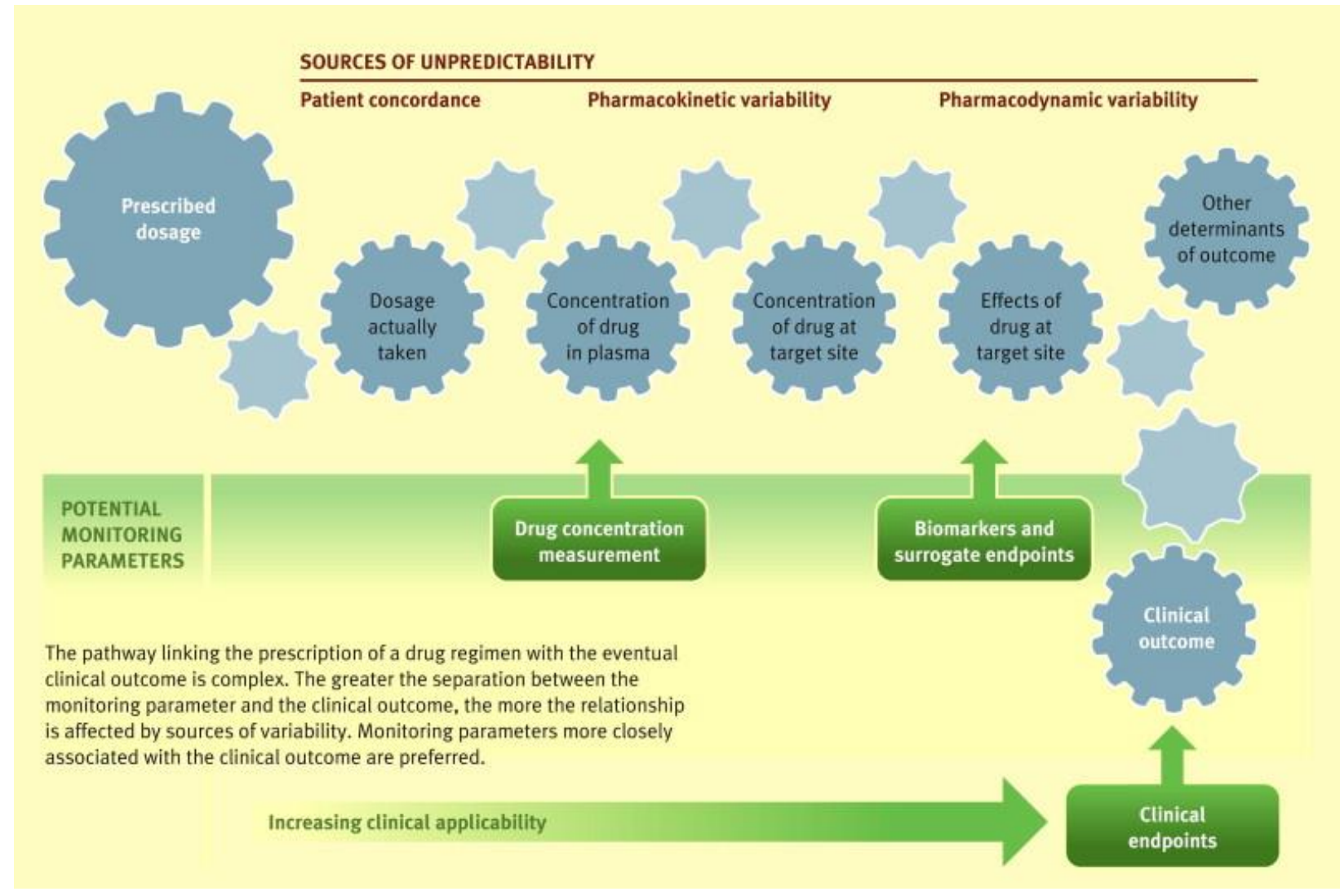




\section{Figure 2}

The semantic distinction between the terms 'biomarker' and 'surrogate endpoint'

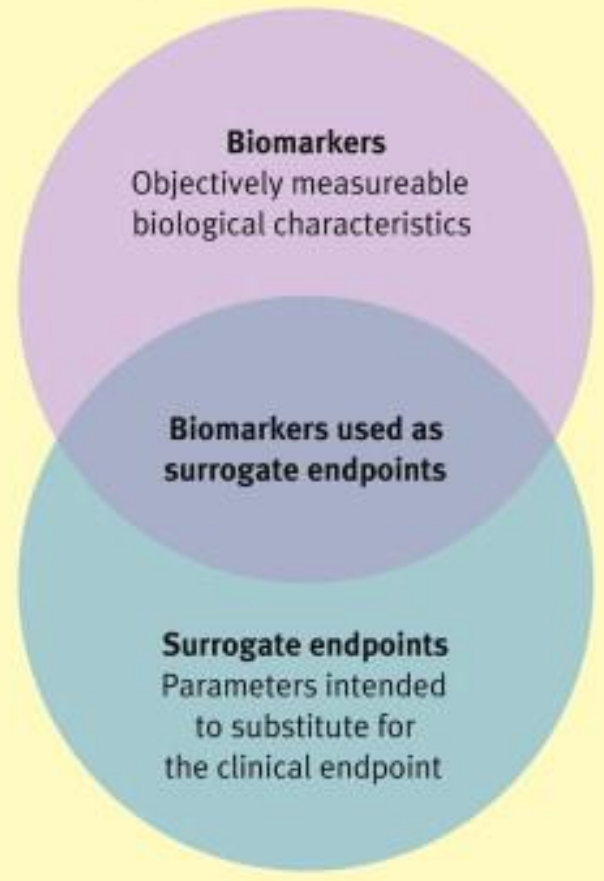




\section{Figure 3}

The factors that may complicate the interpretation of drug concentration measurement
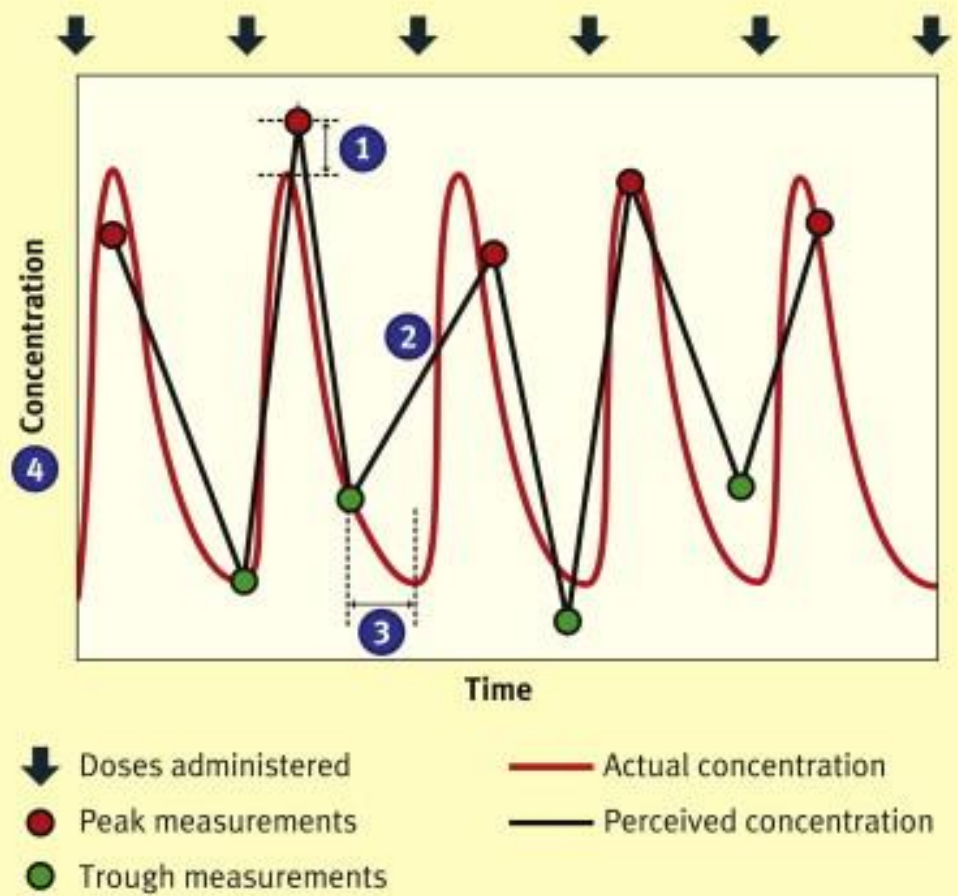

1 Error of the measurement assay

2 Only point measurements are usually taken - the intervening concentrations (which determine overall drug exposure) are not known

(3) Incorrect timing of samples in relation to doses may yield misleading results

(4) Concentration is measured in serum - the concentration at the drug's site of effect is not usually known 

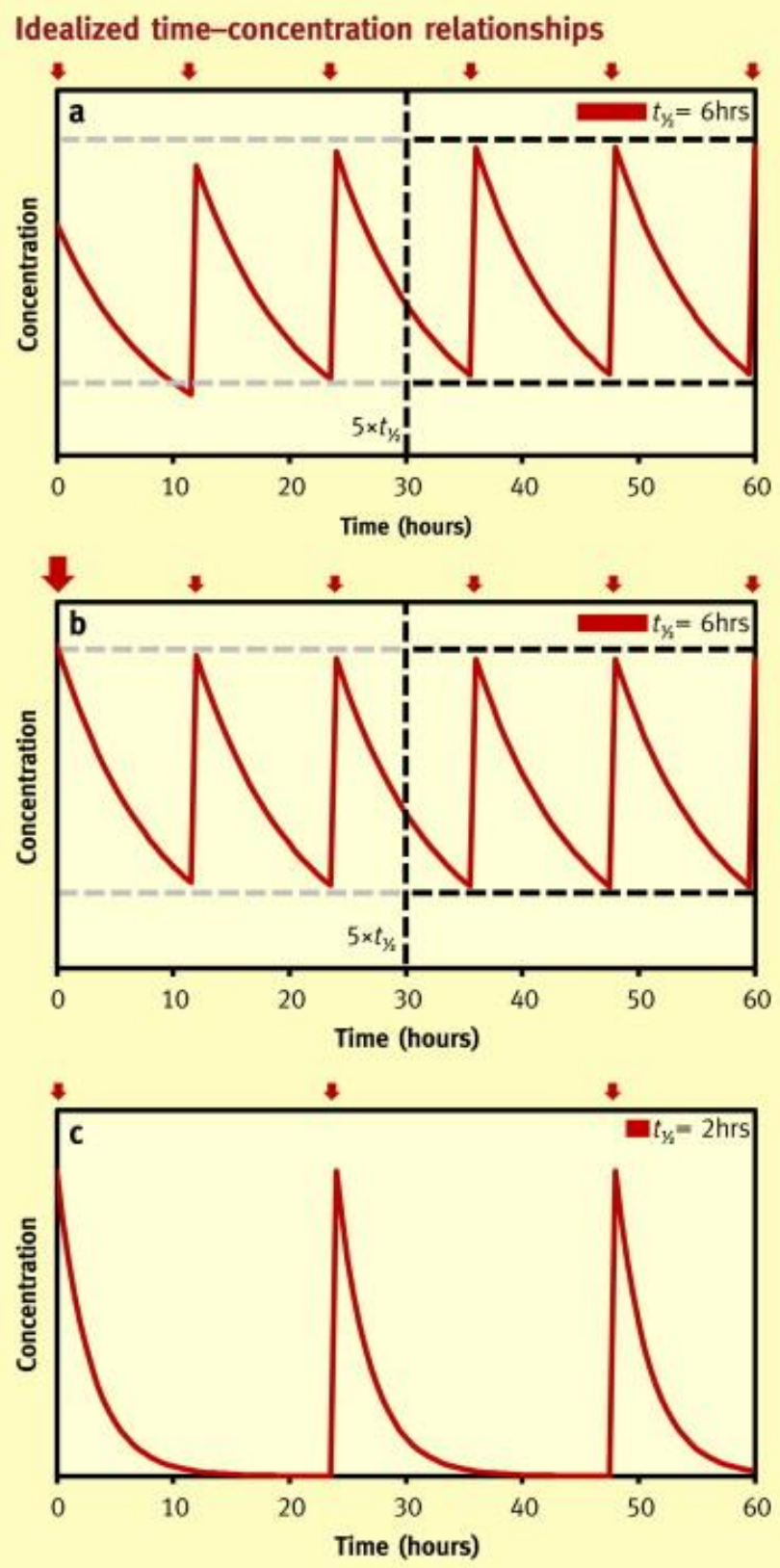

(a): A drug, such as vancomycin, with a half-life of 6 hours, administered intravenously every 12 hours (doses are indicated by arrows). Steady state, indicated by the dashed lines, is attained approximately 5 half-lives $(5 \times 6=30$ hours) after the regimen started. (b) The same drug, this time administered with a loading dose (large arrow). Steady state concentrations are approximated earlier by virtue of the loading dose. (c) A drug, such as gentamicin, with a half-life of 2 hours, administered 24 -hourly. As the dosage interval is substantially longer than the half-life, no appreciable accumulation occurs, such that the concept of steady state is not meaningful.

Figure 4 
Table 1

Drugs commonly subject to plasma concentration monitoring

\begin{tabular}{|c|c|c|c|}
\hline Drug & Half-life $^{a}$ & When to sample & Target concentration \\
\hline Digoxin & 40 hours & $\begin{array}{l}\text { Steady state will be } \\
\text { attained } \\
\text { approximately } 1 \text { week } \\
\text { after starting or } \\
\text { changing a dosage } \\
\text { regimen. Samples } \\
\text { should be taken at } \\
\text { least } 6 \text { hours after a } \\
\text { dose }\end{array}$ & $\begin{array}{l}\text { Efficacy is best determined } \\
\text { with a clinical or surrogate } \\
\text { endpoint (e.g. heart rate). } \\
\text { The risk of toxicity increases } \\
\text { progressively at } \\
\text { concentrations }>1.5 \\
\text { microgram/litre (1.92 } \\
\text { nmol/litre), and becomes } \\
\text { likely at concentrations }>3.0 \\
\text { microgram/litre ( } 3.84 \\
\text { nmol/litre) }\end{array}$ \\
\hline Gentamicin & 2 hours & $\begin{array}{l}\text { Consult local } \\
\text { guidelines. Often, pre- } \\
\text { dose (trough) } \\
\text { concentrations are } \\
\text { measured }\end{array}$ & $\begin{array}{l}\text { Consult local guidelines. } \\
\text { Typically, in once-daily } \\
\text { dosing, trough } \\
\text { concentrations }<1 \\
\text { microgram/litre are targeted }\end{array}$ \\
\hline Lithium & $\begin{array}{l}18-36 \text { hours } \\
\text { (varies between } \\
\text { formulations) }\end{array}$ & $\begin{array}{l}\text { Weekly after initiation } \\
\text { and dosage changes } \\
\text { until concentrations } \\
\text { are stable, then every } \\
3 \text { months. Samples } \\
\text { should be taken } 12 \\
\text { hours after the dose }\end{array}$ & $0.4-1 \mathrm{mmol} /$ litre \\
\hline Phenytoin & $\begin{array}{l}\text { Varies as a } \\
\text { function of } \\
\text { plasma } \\
\text { concentration } \\
\text { (average } 22 \\
\text { hours, range 7- } \\
42 \text { hours) }\end{array}$ & $\begin{array}{l}\text { Acute: } 1 \text { hour after an } \\
\text { intravenous loading } \\
\text { dose to aid the } \\
\text { determination of } \\
\text { maintenance dose or } \\
\text { need to reload } \\
\text { Chronic: trough } \\
\text { concentrations are } \\
\text { preferable }\end{array}$ & $\begin{array}{l}10-20 \mathrm{mg} / \text { litre }(40-80 \\
\mathrm{micromol} / \mathrm{litre})\end{array}$ \\
\hline
\end{tabular}

\section{Notes}

The BNF does not recommend routine monitoring of serum digoxin

concentrations, although it suggests it may be useful to confirm a clinical impression of toxicity or nonadherence. Toxicity can occur even when the concentration is below the 'toxic threshold', particularly with hypokalaemia

Serum concentration measurement is essential in parenteral aminoglycoside therapy. There are no nationally accepted monitoring guidelines for once-daily dosing; local protocols should be consulted

The BNF recommends that lithium should not be prescribed unless facilities for monitoring serum lithium concentrations are available

The BNF recommends monitoring serum phenytoin concentration to guide dosage adjustment. There is a good relationship between plasma concentration and clinical effects In the presence of hypoalbuminaemia, the free (unbound) phenytoin concentration should ideally be measured. If this is facility is not 


\begin{tabular}{|c|c|c|c|c|}
\hline Drug & Half-life ${ }^{a}$ & When to sample & Target concentration & Notes \\
\hline & & & & $\begin{array}{l}\text { available, a correction can be applied } \\
\text { to allow the total concentration to be } \\
\text { assessed against the usual target } \\
\text { range. A formula proposed for the } \\
\text { correction of serum phenytoin } \\
\text { concentrations in the elderly, critically } \\
\text { ill and head-injured patients is: } \\
C_{\text {adj }}=\text { Cobs }^{\prime} \div\left[\left(0.025 \times \mathrm{C}_{\text {alb }}+0.1\right)\right] \\
\text { Where: } \\
\mathrm{C}_{\text {adj }}=\text { adjusted phenytoin concentration } \\
\text { (mg/litre) } \\
\mathrm{C}_{\text {obs }}=\text { observed phenytoin } \\
\text { concentration (mg/litre) } \\
\mathrm{C}_{\text {alb }}=\text { serum albumin concentration } \\
\text { (g/litre) }\end{array}$ \\
\hline $\begin{array}{l}\text { Theophylline } \\
\text { (and } \\
\text { aminophylline) }\end{array}$ & 3-9 hours & $\begin{array}{l}\text { At least } 5 \text { days after } \\
\text { starting treatment or } \\
\text { dosage changes. } \\
\text { Samples should be } \\
\text { taken } 4-6 \text { hours after } \\
\text { the dose }\end{array}$ & $\begin{array}{l}10-20 \mathrm{mg} / \text { litre }(55-110 \\
\mathrm{micromol} / \text { litre) }\end{array}$ & $\begin{array}{l}\text { Monitoring plasma theophylline } \\
\text { concentrations is recommended, } \\
\text { although it is noted that some patients } \\
\text { can achieve sufficient bronchodilation } \\
\text { at concentrations below the target } \\
\text { range; likewise, adverse effects can } \\
\text { occur within the target range }\end{array}$ \\
\hline Vancomycin & 6 hours & $\begin{array}{l}\text { A pre-dose (trough) } \\
\text { concentration should } \\
\text { be taken after } 3-4 \\
\text { doses have been } \\
\text { administered }\end{array}$ & $\begin{array}{l}10-15 \mathrm{mg} / \text { /itre }(15-20 \\
\mathrm{mg} / \text { /itre for severe infections } \\
\text { or less sensitive organisms) }\end{array}$ & $\begin{array}{l}\text { The BNF recommends that plasma } \\
\text { concentration monitoring is required }\end{array}$ \\
\hline
\end{tabular}

BNF, British National Formulary.

aTypical elimination half-lives in adults. Depending on the drug, the half-life may be altered in renal or hepatic impairment, at extremes of age and by the effect of concomitant drugs. See the BNF or the drugs' summaries of product characteristics for details (available at www.medicines.org.uk). 


\section{KEY REFERENCES}

1 Biomarkers Definitions Working Group. Biomarkers and surrogate endpoints: preferred definitions and conceptual framework. Clin Pharmacol Ther 2001; 69: 89-95.

2 Aronson JK, Hardman M. Measuring plasma drug concentrations. Br Med J 1992; 305: 1078-80.

3 Gross AS. Best practice in therapeutic drug monitoring. Br J Clin Pharmacol 2001; 52: 5S-10.

4 Anderson GD, Pak C, Doane KW, et al. Revised Winter-Tozer equation for normalised phenytoin concentrations in trauma and elderly patients with hypoalbuminemia. Ann Pharmacother 1997; 31: 27984.

5 Glasziou PP, Aronson JK. An introduction to monitoring therapeutic interventions in clinical practice. In: Glasziou PP, ed. Evidence-based medical monitoring: from principles to practice. Oxford: Blackwell

Publishing, 2008. pp. 3-14. 